\title{
Different phosphorylation rates among vertebrate cone visual pigments with different spectral sensitivities.
}

\section{$\operatorname{AUTHOR}(\mathrm{S})$ :}

Tsutsui, Kei; Tachibanaki, Shuji; ShimauchiMatsukawa, Yoshie; Shichida, Yoshinori; Kawamura, Satoru

\section{CITATION:}

Tsutsui, Kei ... [et al]. Different phosphorylation rates among vertebrate cone visual pigments with different spectral sensitivities.. Biochemical and biophysical research communications 2013, 440(4): 630-634

\section{ISSUE DATE:}

2013-11-01

URL:

http://hdl.handle.net/2433/179767

\section{RIGHT:}

@ 2013 Elsevier Inc.; この論文は著者最終稿です。内容が印刷版と異な ることがありますので、引用の際には出版社版をご確認ご利用くださ $\omega_{0}$ This is the Accepted Author Manuscript. Please cite only the published version. 


\section{Different Phosphorylation Rates among Vertebrate Cone Visual Pigments with Different Spectral Sensitivities}

Kei Tsutsui, ${ }^{\dagger},{ }^{*}, 1$ Shuji Tachibanaki, ${ }^{\dagger}$ Yoshie Shimauchi-Matsukawa ${ }^{\dagger}$ Yoshinori Shichida, ${ }^{\dagger}$ and Satoru Kawamura ${ }^{\dagger}$

${ }^{\dagger}$ Graduate School of Frontier Biosciences, Osaka University, 1-3 Yamadaoka, Suita, Osaka 5650871, Japan

${ }^{\ddagger}$ Department of Biophysics, Graduate School of Science, Kyoto University, KitashirakawaOiwakecho, Sakyo-ku, Kyoto 606-8502, Japan

* Corresponding author

Primate Research Institute, Kyoto University, 41-2 Kanrin, Inuyama, Aichi 484-8506, Japan E-mail: tsutsui.kei.4c@kyoto-u.ac.jp

Tel: +81-0568-63-0580

${ }^{1}$ Present Address: Primate Research Institute, Kyoto University, 41-2 Kanrin, Inuyama, Aichi 484-8506, Japan 


\begin{abstract}
Cone photoreceptor subtypes having different spectral sensitivities exhibit different recovery kinetics in their photoresponses in some vertebrates. Phosphorylation by G protein-coupled receptor kinase (GRK) is essential for the rapid inactivation of light-activated visual pigment, which is the rate-limiting step of the cone photoresponse recovery in salamander. In this study we compared the rate of light-dependent phosphorylation by GRK7 of carp green- and bluesensitive cone visual pigments. Blue pigment was phosphorylated significantly less effectively than green pigment, suggesting that the difference in the pigment phosphorylation rate is responsible for the difference in photoresponse kinetics among cone photoreceptor subtypes.
\end{abstract}

Keywords: cone visual pigment, phosphorylation, GRK

\author{
Abbreviations \\ $\lambda_{\text {max }}$, absorption maximum wavelength; GRK, G protein-coupled receptor kinase; UV, \\ ultraviolet; CHAPS, 3-[(3-cholamidopropyl)dimethylammonio]-1-propanesulfonate; PC, L- $\alpha$ - \\ phosphatidylcholine; Pi, inorganic phosphate.
}




\section{Introduction}

Vertebrate eyes have two types of photoreceptor cells, rods and cones. Rods mediate night vision and cones mediate daylight and color visions. Functional differences between rods and cones and their molecular mechanisms have been extensively investigated [1]. Although less studied, it is known that cone subtypes having different spectral sensitivities differ in their other properties, such as response sensitivity and kinetics. For example, it is reported that cone photoreceptors exhibit different kinetics of photoresponse recovery in amphibian [2] and fish $[3,4]$ species. However, it is largely unknown what causes these differences.

Rod and cone photoreceptors contain visual pigments. Vertebrate visual pigments are classified into five groups, that is, four cone pigment groups [L (LWS), S (SWS1), M1 (SWS2), M2 (Rh2)] and one rhodopsin group [Rh (Rh1)], on the basis of amino acid sequence similarity [5-7]. This classification is well correlated with their absorption maximum wavelength $\left(\lambda_{\max }\right)$. It is well known that rhodopsin and cone pigments differ in some functional properties such as protein stability, photoreaction kinetics and regeneration rate [8]. On the other hand, differences in properties other than absorption wavelength among L, S, M1 and M2 groups are less investigated [8-10].

Phosphorylation of visual pigment by G protein-coupled receptor kinase (GRK) and subsequent binding of arrestin to the phosphorylated pigment are essential steps for the inactivation of photoactivated visual pigment and hence for the termination of phototransduction [11]. Recently, it was shown that the inactivation of the photoactivated visual pigment is the ratelimiting step of recovery of photoresponse in salamander cones at least for saturating responses $[12,13]$. This made us speculate that different rates of phosphorylation between cone pigments 
with different spectral sensitivities might be responsible for the difference in photoresponse recovery kinetics among cone subtypes.

In previous studies, light-dependent phosphorylation of cone visual pigments were biochemically observed with lizard [14], chicken [15], fish [16-18], and mammalian [19,20] cone pigments. However, to our knowledge, direct comparison of phosphorylation rates between cone pigments with different spectral sensitivities has never been reported.

In our previous studies we performed visual pigment phosphorylation assays with purified carp rods and cones $[16,17]$. However, it is difficult to purify separately cones with different spectral sensitivities. So in the present study, we prepared carp cone visual pigments by expression in HEK293T cells and compared their light-dependent phosphorylation rates by carp GRK7, which is specifically expressed in cones [17,21]. 


\section{Materials and Methods}

\section{Expression and purification of visual pigments}

Red, green 1, green 2, blue and UV opsins (GenBank accession numbers are AB055656, AB110602, AB110603, AB113668 and AB113669, respectively) were cloned from the carp (Cyprinus carpio) retinal cDNA library [21]. The cloned cDNAs of carp cone opsins were tagged by the bovine rhodopsin 1D4 epitope sequence (ETSQVAPA) [22] at their C-terminal, unless noted otherwise. Then the cDNAs were introduced into an expression vector pCAGGS [23]. Opsins were expressed in the HEK293T cell by the calcium-phosphate method as previously reported [24]. After incubation of the transfected cells for 2 days, they were collected by centrifugation and suspended in buffer A (50 mM HEPES, $140 \mathrm{mM} \mathrm{NaCl}, 3 \mathrm{mM} \mathrm{MgCl}$, $\mathrm{pH}$ 6.5). HEK293T cells expressing opsins were supplemented with 11-cis-retinal (final concentration: $20 \mu \mathrm{M}$ ) and incubated overnight to reconstitute the visual pigments. The following procedures were carried out under dim red light. The cell membranes were solubilized in buffer B (0.75\% CHAPS, 10 mM HEPES, $115 \mathrm{mM} \mathrm{K-gluconate,} 2.5 \mathrm{mM} \mathrm{KCl,} 2 \mathrm{mM} \mathrm{MgCl}$, $0.2 \mathrm{mM}$ EGTA, $0.1 \mathrm{mM} \mathrm{CaCl}_{2}, 1 \mathrm{mM}$ DTT, $\mathrm{pH}$ 7.5), and visual pigments were purified by adsorption on an anti-1D4-antibody-conjugated column and elution with buffer B containing 0.3 $\mathrm{mg} / \mathrm{mL} 1 \mathrm{D} 4$ peptide.

\section{Spectrophotometry}

Absorption spectra of visual pigments were recorded at $4^{\circ} \mathrm{C}$ by a Shimadzu UV2400 spectrophotometer.

\section{Preparation of GRK7-expressing cell membrane}

The cDNA of carp GRK7-1a (GenBank accession number is AB055658) [21] was introduced into an expression vector pCAGGS. GRK7 was expressed in the HEK293T cell by 
the calcium-phosphate method. Mevalonolactone was added to the culture medium (final concentration: $3 \mathrm{mM}$ ) right after the transfection in order to facilitate the C-terminal geranylgeranylation of GRK7. After incubation of the transfected cells for 2 days, they were collected by centrifugation and suspended in buffer A. GRK7-containing cell membranes were prepared by sucrose flotation as previously described [25] with some modifications. Briefly, the collected cells were homogenized in buffer A containing $8.6 \%(\mathrm{w} / \mathrm{v})$ sucrose by using a homogenizer, and then layered on top of buffer A containing $40 \%(\mathrm{w} / \mathrm{v})$ sucrose, followed by centrifugation in a swing-bucket rotor at $186,000 \times \mathrm{g}$ for $1 \mathrm{~h}$. The membranes floating at the interface between $8.6 \%$ and $40 \%$ sucrose layers were collected. The collected membranes were suspended in buffer C [10 mM HEPES, $115 \mathrm{mM} \mathrm{K-gluconate,} 2.5 \mathrm{mM} \mathrm{KCl,} 2 \mathrm{mM} \mathrm{MgCl} 2,0.2$ mM EGTA, $0.1 \mathrm{mM} \mathrm{CaCl}_{2}, 1 \mathrm{mM}$ DTT, $\mathrm{pH}$ 7.5]. The amount of GRK7 in the membrane was quantified by SDS-PAGE and Coomassie blue staining with bovine serum albumin as the standard.

\section{Reconstitution of visual pigments into liposome}

Proteoliposomes containing visual pigments were prepared according to the previously reported method [26] with some modifications. Briefly, visual pigments were supplemented with a 500-fold molar excess of L- $\alpha$-phosphatidylcholine (PC) dissolved in buffer B and incubated overnight. Then the samples were rapidly diluted by 5 fold with buffer $\mathrm{C}$ so that the CHAPS concentration was lowered to $0.15 \%$, which was sufficiently lower than the critical micelle concentration $(0.49 \%)$. The remaining monomeric CHAPS was removed by dialysis against a 20-fold excess volume of buffer $\mathrm{C}$ for $\sim 24 \mathrm{~h}$ with four buffer exchanges. PC liposomes were collected by centrifugation at $100,000 \times \mathrm{g}$ for $60 \mathrm{~min}$ and resuspended in buffer $\mathrm{C}$. The aliquots of the liposome suspension were solubilized in buffer B and used for quantification of visual 
pigments in the liposomes by spectrophotometry. Visual pigment concentrations were calculated using the molar extinction coefficient of $40000 \mathrm{M}^{-1} \mathrm{~cm}^{-1}$ at the absorption maximum wavelength.

\section{Phosphorylation Assays}

HEK293T cell membranes expressing GRK7 and PC liposomes containing visual pigment were mixed at an equivalent GRK:pigment molar ratio, frozen at $-80^{\circ} \mathrm{C}$ and then thawed to fuse the membranes right before the phosphorylation assays. Phosphorylation assays were performed at room temperature using a calibrated rapid-quench apparatus in which the timing of the addition of the reaction-stopping solution was controlled, as previously reported [17]. Fused membrane containing 12 pmol of visual pigment and 12 pmol of GRK7 (suspended in $15 \mu \mathrm{l}$ of buffer C) were mixed with $10 \mu \mathrm{l}$ of buffer $\mathrm{C}$ containing $2.5 \mathrm{mM}$ ATP with tracer $\left[\gamma_{-}{ }^{32} \mathrm{P}\right] \mathrm{ATP}$, $1.25 \mathrm{mM}$ GTP and additional $1.5 \mathrm{mM}$ EGTA so that the mixture $(25 \mu \mathrm{l})$ contained $1 \mathrm{mM}$ ATP with tracer $\left[\gamma_{-}{ }^{32} \mathrm{P}\right] \mathrm{ATP}, 0.5 \mathrm{mM}$ GTP, $0.8 \mathrm{mM}$ EGTA, $115 \mathrm{mM} \mathrm{K}$-gluconate, $2.5 \mathrm{mM} \mathrm{KCl}, 2$ $\mathrm{mM} \mathrm{MgCl} 2,0.1 \mathrm{mM} \mathrm{CaCl}_{2}, 10 \mathrm{mM}$ HEPES and $1 \mathrm{mM}$ DTT. After preincubation for $30 \mathrm{sec}$, the sample was irradiated with a white light flash which bleached $\sim 50 \%$ of the pigment. The flash illumination was omitted for "dark" samples. The reaction was terminated at $0,0.1,0.2,0.3,0.5$, 0.75 or $1 \mathrm{sec}$ after the flash by adding $\sim 150 \mu \mathrm{l}$ of $10 \%(\mathrm{w} / \mathrm{v})$ trichloroacetic acid. After centrifugation $(20,000 \times \mathrm{g}$ for $1 \mathrm{~h})$, the precipitate was washed with buffer $\mathrm{C}$ without DTT and subjected to SDS-PAGE. Then the amount of ${ }^{32} \mathrm{P}$ incorporated into the visual pigment band was quantified. The data of "dark" samples were subtracted from the data of "light" samples to calculate the light-dependent phosphorylation.

Phosphorylation assays of P189V mutants of green 1 and blue pigments were performed similarly, except that the reaction was terminated at $10 \mathrm{sec}$ after the flash. 


\section{Results}

\section{Expression of carp cone visual pigments}

First, we cloned carp (Cyprinus carpio) red (L group), green 1 (M2 group), green 2 (M2 group), blue (M1 group) and UV (S group) cone visual pigments from retinal cDNA library [21] (GenBank accession numbers are AB055656, AB110602, AB110603, AB113668 and AB113669, respectively). Then we tagged them with the 1D4 epitope sequence (ETSQVAPA) at the Cterminal, cloned them into an expression vector and expressed in HEK293T cells. The pigment in transfected HEK293T cell membranes was regenerated with 11-cis-retinal, solubilized with CHAPS and affinity-purified by the 1D4 antibody. Bovine rhodopsin was also expressed, regenerated and purified as a control.

Absorption spectra of the purified expressed carp cone visual pigments and bovine rhodopsin are shown in Figure 1. Green 1, green 2, blue and UV pigments exhibited their $\lambda_{\max }$ values at $510 \mathrm{~nm}, 500 \mathrm{~nm}, 445 \mathrm{~nm}$ and $370 \mathrm{~nm}$, respectively. These values are in good agreement with the reported values for goldfish pigments (green1: $511 \mathrm{~nm}$, green2: $506 \mathrm{~nm}$, blue: $440 \mathrm{~nm}, \mathrm{UV}: 359 \mathrm{~nm}$ ) [27,28] which have 92-97\% amino acid sequence identities to those of carp pigments. Unfortunately, the expression level of red pigment was not sufficient for obtaining a reliable absorption spectrum.

It should be noted that native carp visual pigments contain 11-cis 3,4-dehydroretinal (A2 11-cis-retinal), not A1 11-cis-retinal. Absorption spectra of carp cone visual pigments extracted from native carp photoreceptor membranes are known ( $\lambda_{\max }$ values; red: $618 \mathrm{~nm}$, green: $535 \mathrm{~nm}$, blue: $460 \mathrm{~nm}$ ) [16]. Absorption maximum of native carp UV pigment determined by microspectrophotometry is $377.5 \mathrm{~nm}$ [29]. An empirical relationship between the $\lambda_{\max }$ of a visual pigment and the $\lambda_{\max }$-shift induced by an A1/A2 chromophore exchange is known [30]. 
We used this relationship to predict the $\lambda_{\max }$ of an A2 carp cone visual pigment from the observed $\lambda_{\max }$ of an expressed A1 pigment: green1: $540 \mathrm{~nm}$, green2: $524 \mathrm{~nm}$, blue: $453 \mathrm{~nm}, \mathrm{UV}$ : $385 \mathrm{~nm}$. These are in good agreement with values from native A2 pigments noted above.

\section{Phosphorylation rates of cone visual pigments by GRK7}

We expressed carp GRK7 (GenBank accession number: AB055658) [21], which is specifically expressed in cones [17], in HEK293T cells, and prepared a cell membrane preparation expressing GRK7 by purification with sucrose floatation.

Purified pigments were reconstituted into phosphatidylcholine liposomes and they were fused with GRK7-expressing HEK293T cell membranes. Then the rates of light-dependent phosphorylation by GRK7 of green 1 pigment, blue pigment and bovine rhodopsin were measured at room temperature. A rapid-quench apparatus [17] was used in order to observe the early time course $(<1 \mathrm{sec})$ of phosphorylation, because the lifetimes of photoactivated cone visual pigments at room temperature are in this range [8]. Phosphorylation reaction was started with a white flash (which bleaches $\sim 50 \%$ of the pigment) and quenched by adding a denaturing solution at various time delays. Dark phosphorylation was subtracted from the data to evaluate light-dependent phosphorylation.

Figure 2A shows the time courses of light-dependent phosphorylation of green 1 pigment, blue pigment and bovine rhodopsin by GRK7, which were averaged over four independent experiments. Figure $2 \mathrm{~B}$ shows the summarized results of the phosphorylation rates obtained by fitting the data with a straight line in the linear range of the signal $(0-0.5 \mathrm{sec})$ in each experiment. Blue pigment was phosphorylated significantly (pairwise t-test, $p<0.05$ ) less effectively than green pigment (the average values were different by $\sim 2$-fold), whereas bovine rhodopsin was phosphorylated as effectively as green pigment. 


\section{Phosphorylation of visual pigments with no $1 D 4$ sequence attached}

It should be noted that we used pigments tagged with the 1D4 epitope sequence (ETSQVAPA) at the C-terminal for the purpose of affinity purification. In order to exclude the possibility that addition of the 1D4 sequence affected the difference in the phosphorylation rates between green 1 and blue pigments, we first tried to perform phosphorylation assays with HEK293T cell membranes expressing pigments with no 1D4 sequence attached. However nonspecific background phosphorylation was too high and the signal-to-noise ratio was low (data not shown). In order to improve this, we introduced P189V mutation (bovine rhodopsin numbering system) in green 1 and blue pigments. A similar mutation P189I is reported to confer a higher expression level and a longer-lived ( 26 fold) active intermediate in a cone pigment [31], with the former increasing the signal by elevating the amount of visual pigments expressed in the cell membrane and the latter increasing the signal by enabling the timing of reaction termination later than $1 \mathrm{sec}$. We successfully measured phosphorylation rates of the P189V mutants in HEK293T cell membranes. The phosphorylation level of the P189V mutant of blue pigment at $10 \mathrm{sec}$ after irradiation was significantly lower than that of the P189V mutant of green 1 pigment (Figure 3), suggesting that the addition of the 1D4 sequence does not affect the difference in the phosphorylation rate found between green 1 and blue pigments. 


\section{Discussion}

In this study, we show that carp blue pigment is phosphorylated by GRK7 less efficiently than green pigment. To our knowledge, this is the first demonstration that any cone visual pigments exhibit different rates of phosphorylation by GRK among subtypes.

It is known that photoactivated pigment activates GRK and then activated GRK phosphorylates pigments [32]. Therefore the observed difference in the phosphorylation rate could be due to the difference in GRK activation rate and/or the difference in susceptibility of pigments to phosphorylation. It is not possible from the present data to determine which (or both) is the case.

It is reported that the photoresponse recovery in goldfish green cones is faster than that in blue cones [4]. The amino acid sequences of carp and goldfish cone visual pigments are highly similar (amino acid identity; green 1: 96\%, blue; 95\%). Therefore, it would be reasonable to postulate that the difference in the phosphorylation rate between green and blue pigments found in carp is also present in goldfish. Because inactivation of photoactivated visual pigment is the rate-limiting step in the recovery of bright flash photoresponses in salamander cones $[12,13]$, the postulated faster pigment phosphorylation in goldfish green pigment could be an underlying mechanism of a faster response recovery in goldfish green cones than in blue cones. In striped bass [3] and tiger salamander [2], it has been also reported that photoresponse recovery time courses are different among cones depending on their spectral sensitivities, which might be due to a difference in the rate of visual pigment phosphorylation.

There are also some reports comparing photoresponse kinetics among mammalian cones having different spectral sensitivities. The photoresponse recovery in green cones is reported to be faster than that in red cones in macaque [33] and human [34], although there is a report 
demonstrating that macaque red, green and blue cones show similar photoresponse kinetics [35]. Dim flash photoresponses in mouse green and UV cones show similar recovery kinetics [36]. It will be interesting to compare the phosphorylation rates in mammalian visual pigments.

In summary, we expressed carp green 1, green 2, blue and UV cone visual pigments, and their absorption spectra were measured. Then the rates of light-dependent phosphorylation of green 1 and blue pigments by GRK7 were compared. The phosphorylation rate for blue pigment was significantly lower than that for green pigment. This result, together with the previously reported difference in photoresponse recovery rate between goldfish green and blue cones [4], suggests that the difference in the photoresponse recovery rate among cone photoreceptors having different spectral sensitivities is due to the difference in the phosphorylation rate of visual pigments. 


\section{Acknowledgements}

We thank Prof. R. S. Molday for the generous gift of a Rho1D4-producing hybridoma and Prof. H. Niwa for pCAGGS vector. This work was supported by the Japan Society for the Promotion of Science Grants 23227002 (to S. K.) and by the Japanese Society for the Promotion of Science Research Fellowships for Young Scientists (to K.T.). 


\section{References}

[1] S. Kawamura, S. Tachibanaki, Rod and cone photoreceptors: molecular basis of the difference in their physiology, Comp. Biochem. Physiol. A Mol. Integr. Physiol. 150 (2008) 369-377.

[2] R.J. Perry, P.A. McNaughton, Response properties of cones from the retina of the tiger salamander, J. Physiol. 433 (1991) 561-587.

[3] J.L. Miller, J.I. Korenbrot, Phototransduction and adaptation in rods, single cones, and twin cones of the striped bass retina: a comparative study, Vis. Neurosci. 10 (1993) 653-667.

[4] A.G. Palacios, F.J. Varela, R. Srivastava, T.H. Goldsmith, Spectral sensitivity of cones in the goldfish, Carassius auratus, Vision Res. 38 (1998) 2135-2146.

[5] T. Okano, D. Kojima, Y. Fukada, Y. Shichida, T. Yoshizawa, Primary structures of chicken cone visual pigments: vertebrate rhodopsins have evolved out of cone visual pigments, Proc. Natl. Acad. Sci. U.S.A. 89 (1992) 5932-5936.

[6] S. Yokoyama, Molecular evolution of vertebrate visual pigments, Prog. Retin. Eye Res. 19 (2000) 385-419.

[7] T. Ebrey, Y. Koutalos, Vertebrate photoreceptors, Prog. Retin. Eye Res. 20 (2001) 49-94.

[8] H. Imai, S. Kuwayama, A. Onishi, T. Morizumi, O. Chisaka, Y. Shichida, Molecular properties of rod and cone visual pigments from purified chicken cone pigments to mouse rhodopsin in situ, Photochem. Photobiol. Sci. 4 (2005) 667-674.

[9] B. Nickle, P.R. Robinson, The opsins of the vertebrate retina: insights from structural, biochemical, and evolutionary studies, Cell. Mol. Life Sci. 64 (2007) 2917-2932. 
[10] K. Sato, T. Yamashita, Y. Imamoto, Y. Shichida, Comparative studies on the late bleaching processes of four kinds of cone visual pigments and rod visual pigment, Biochemistry (2012) 51, 4300-4308.

[11] T. Maeda, Y. Imanishi, K. Palczewski, Rhodopsin phosphorylation: 30 years later, Prog. Retin. Eye Res. 22 (2003) 417-434.

[12] H.R. Matthews, A.P. Sampath, Photopigment quenching is $\mathrm{Ca}^{2+}$ dependent and controls response duration in salamander L-cone photoreceptors, J. Gen. Physiol. 135 (2010) 355366.

[13] J. Zang, H.R. Matthews, Origin and control of the dominant time constant of salamander cone photoreceptors, J. Gen. Physiol. 140 (2012) 219-233.

[14] A.E. Walter, T.A. Shuster, D.B. Farber, Light-induced phosphorylation of proteins from the all-cone retina of the lizard, Anolis carolinensis, Invest. Ophthalmol. Vis. Sci. 27 (1986) 1609-1614.

[15] Y. Fukada, K. Kokame, T. Okano, Y. Shichida, T. Yoshizawa, J.H. McDowell, P.A. Hargrave, K. Palczewski, Phosphorylation of iodopsin, chicken red-sensitive cone visual pigment, Biochemistry 29 (1990) 10102-10106.

[16] S. Tachibanaki, S. Tsushima, S. Kawamura, Low amplification and fast visual pigment phosphorylation as mechanisms characterizing cone photoresponses, Proc. Natl. Acad. Sci. U.S.A. 98 (2001) 14044-14049.

[17] S. Tachibanaki, D. Arinobu, Y. Shimauchi-Matsukawa, S. Tsushima, S. Kawamura, Highly effective phosphorylation by $\mathrm{G}$ protein-coupled receptor kinase 7 of light-activated visual pigment in cones, Proc. Natl. Acad. Sci. U.S.A. 102, (2005) 9329-9334. 
[18] M.J. Kennedy, F.A. Dunn, J.B. Hurley, Visual pigment phosphorylation but not transducin translocation can contribute to light adaptation in zebrafish cones, Neuron 41, (2004) 915928.

[19] X. Zhu, B. Brown, A. Li, A.J. Mears, A. Swaroop, C.M. Craft, GRK1-dependent phosphorylation of $\mathrm{S}$ and $\mathrm{M}$ opsins and their binding to cone arrestin during cone phototransduction in the mouse retina, J. Neurosci. 23 (2003) 6152-6160.

[20] P. Liu, S. Osawa, E.R. Weiss, M opsin phosphorylation in intact mammalian retinas, J. Neurochem. 93 (2005) 135-144.

[21] Y. Shimauchi-Matsukawa, Y. Aman, S. Tachibanaki, S. Kawamura, Isolation and characterization of visual pigment kinase-related genes in carp retina: polyphyly in GRK1 subtypes, GRK1A and 1B, Mol. Vis. 11 (2005) 1220-1228.

[22] D.D. Oprian, A.B. Asenjo, N. Lee, S.L. Pelletier, Design, chemical synthesis, and expression of genes for the three human color vision pigments, Biochemistry 30 (1991) 11367-11372.

[23] H. Niwa, K. Yamamura, J. Miyazaki, Efficient selection for high-expression transfectants with a novel eukaryotic vector, Gene 108 (1991) 193-199.

[24] H. Imai, A. Terakita, Y. Shichida, Analysis of amino acid residues in rhodopsin and cone visual pigments that determine their molecular properties, Methods Enzymol. 315 (2000) 293-312.

[25] D. Kojima, T. Oura, O. Hisatomi, F. Tokunaga, Y. Fukada, T. Yoshizawa, Y. Shichida, Molecular properties of chimerical mutants of gecko blue and bovine rhodopsin, Biochemistry 35 (1996) 2625-2629. 
[26] S.L. Niu, B. Doctrow, D.C. Mitchell, Rhodopsin activity varies in proteoliposomes prepared by different techniques, Biochemistry 48 (2009) 156-163.

[27] R.L. Johnson, K.B. Grant, T.C. Zankel, M.F. Boehm, S.L. Merbs, J. Nathans, K. Nakanishi, Cloning and expression of goldfish opsin sequences, Biochemistry 32 (1993) 208-214.

[28] S. Yokoyama, F.B. Radlwimmer, S. Kawamura, Regeneration of ultraviolet pigments of vertebrates, FEBS Lett. 423 (1998) 155-158.

[29] C.W. Hawryshyn, F.I. Hárosi, Ultraviolet photoreception in carp: microspectrophotometry and behaviorally determined action spectra, Vision Res. 31 (1991) 567-576.

[30] F.I. Hárosi, An analysis of two spectral properties of vertebrate visual pigments, Vision Res. 34 (1994) 1359-1367.

[31] S. Kuwayama, H. Imai, T. Hirano, A. Terakita, Y. Shichida, Conserved proline residue at position 189 in cone visual pigments as a determinant of molecular properties different from rhodopsins, Biochemistry 41 (2002) 15245-15252.

[32] N.G. Brown, C. Fowles, R. Sharma, M. Akhtar, Mechanistic studies on rhodopsin kinase. Light-dependent phosphorylation of C-terminal peptides of rhodopsin, Eur. J. Biochem. 208 (1992) 659-667.

[33] B.J. Nunn,J.L. Schnapf, D.A. Baylor, Spectral sensitivity of single cones in the retina of Macaca fascicularis, Nature 309 (1984) 264-266.

[34] J.L. Schnapf, T.W. Kraft, D.A. Baylor, Spectral sensitivity of human cone photoreceptors, Nature 325 (1987) 439-441.

[35] J.L. Schnapf, B.J. Nunn, M. Meister, D.A. Baylor, Visual transduction in cones of the monkey Macaca fascicularis, J. Physiol. 427 (1990) 681-713. 
[36] S.S. Nikonov, B.M. Brown, J.A. Davis, F.I. Zuniga, A. Bragin, E.N. Pugh Jr., C.M. Craft, Mouse cones require an arrestin for normal inactivation of phototransduction, Neuron 59 (2008) 462-474. 


\section{Figure Legends}

Figure 1. Absorption Spectra of carp cone visual pigments and bovine rhodopsin. (A) Green 1 pigment. (B) Green 2 pigment. (C) Blue pigment. (D) UV pigment. (E) Bovine rhodopsin. The absorption maximum wavelength is indicted in each panel.

Figure 2. Light-dependent phosphorylation of carp green 1 and blue pigments by GRK7. (A) Time courses of light-dependent Pi incorporation in visual pigments. Data are shown as a mean \pm standard deviation obtained in four independent experiments. Solid circles stands for the results obtained in green 1 pigment, solid triangles in blue pigment, and open squares in bovine rhodopsin. (B) Phosphorylation rates determined by fitting the data in (A) with straight lines in the linear range of the signal $(0-0.5 \mathrm{sec}) . \mathrm{G}$ stands for the result obtained in green 1 pigment, B in blue pigment and $\mathrm{bRh}$ in bovine rhodopsin. Error bars represent standard deviations. The asterisk represents a significant difference (pairwise t-test, $p<0.05$ ).

Figure 3. Light-dependent phosphorylation by GRK7 of the P189V mutants of carp green 1 and blue pigments with no 1D4 sequence attached. Light-dependent phosphorylation levels at $10 \mathrm{sec}$ after irradiation are expressed as relative values to that of wild-type bovine rhodopsin. $\mathrm{G}$ stands for the result obtained in the P189V mutant of green 1 pigment and B in the P189V mutant of blue pigment. Error bars represent standard deviations. The asterisk represents a significant difference (t-test, $p<0.05)$. 


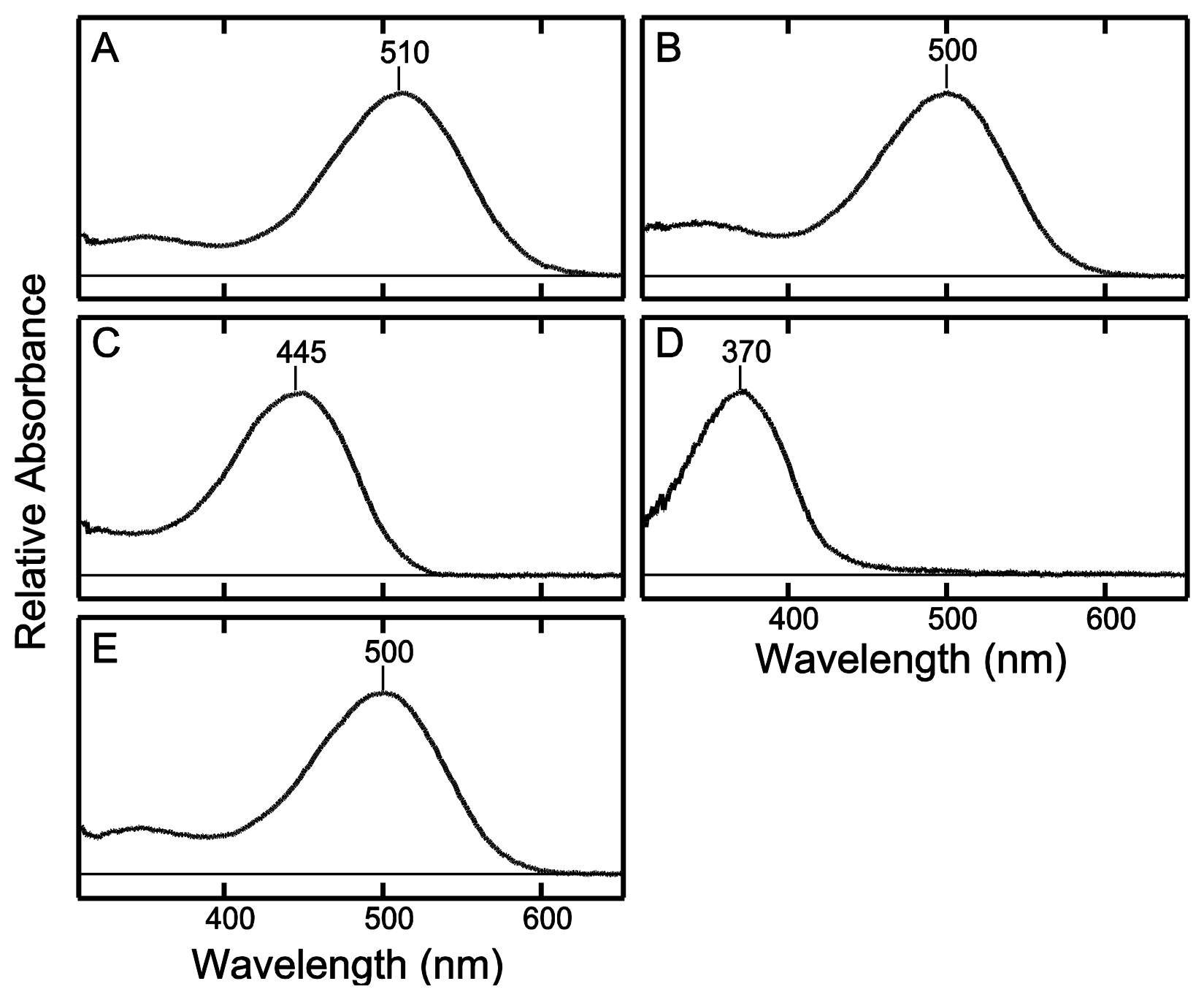

Figure 1. 
A

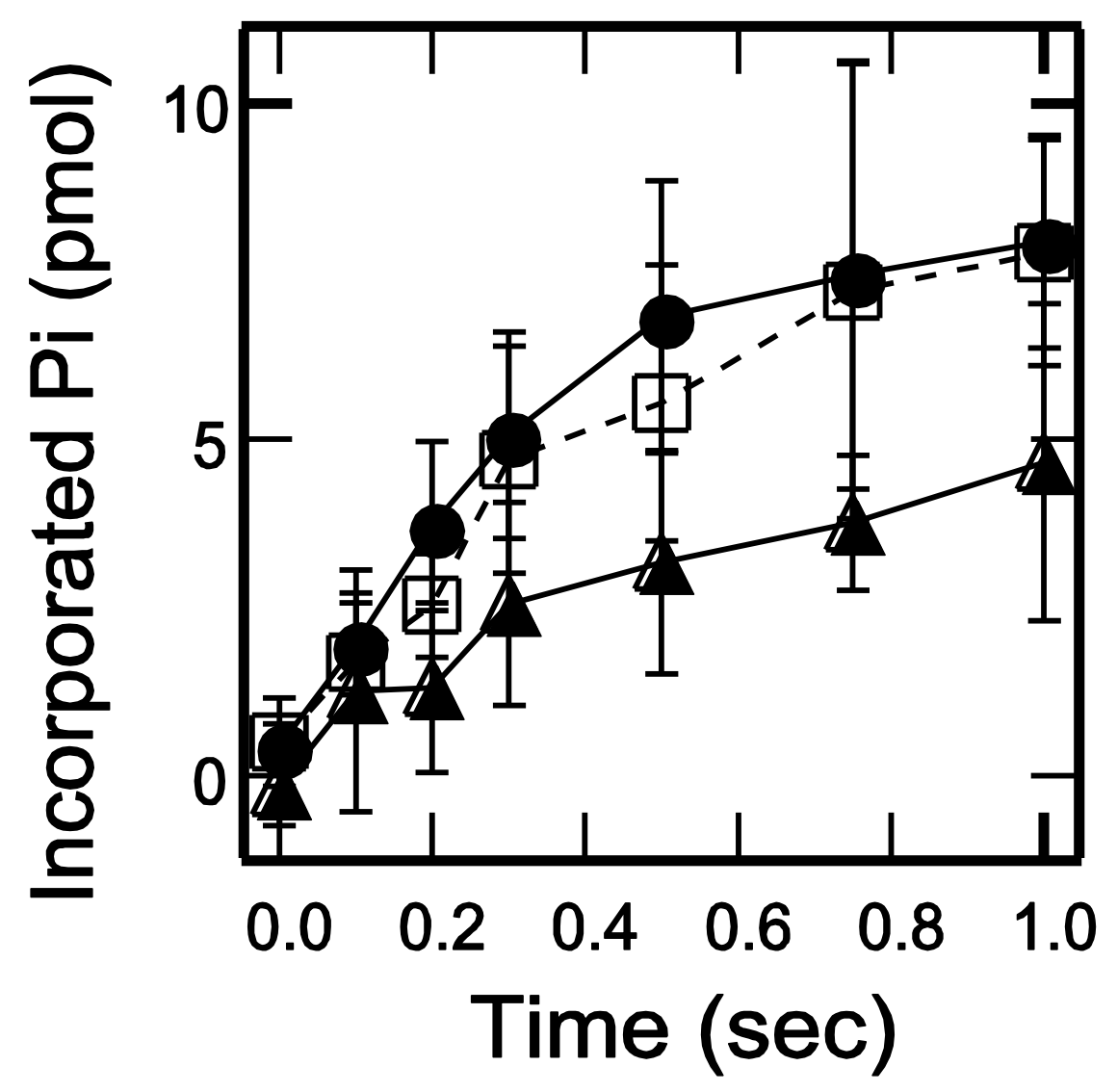

B

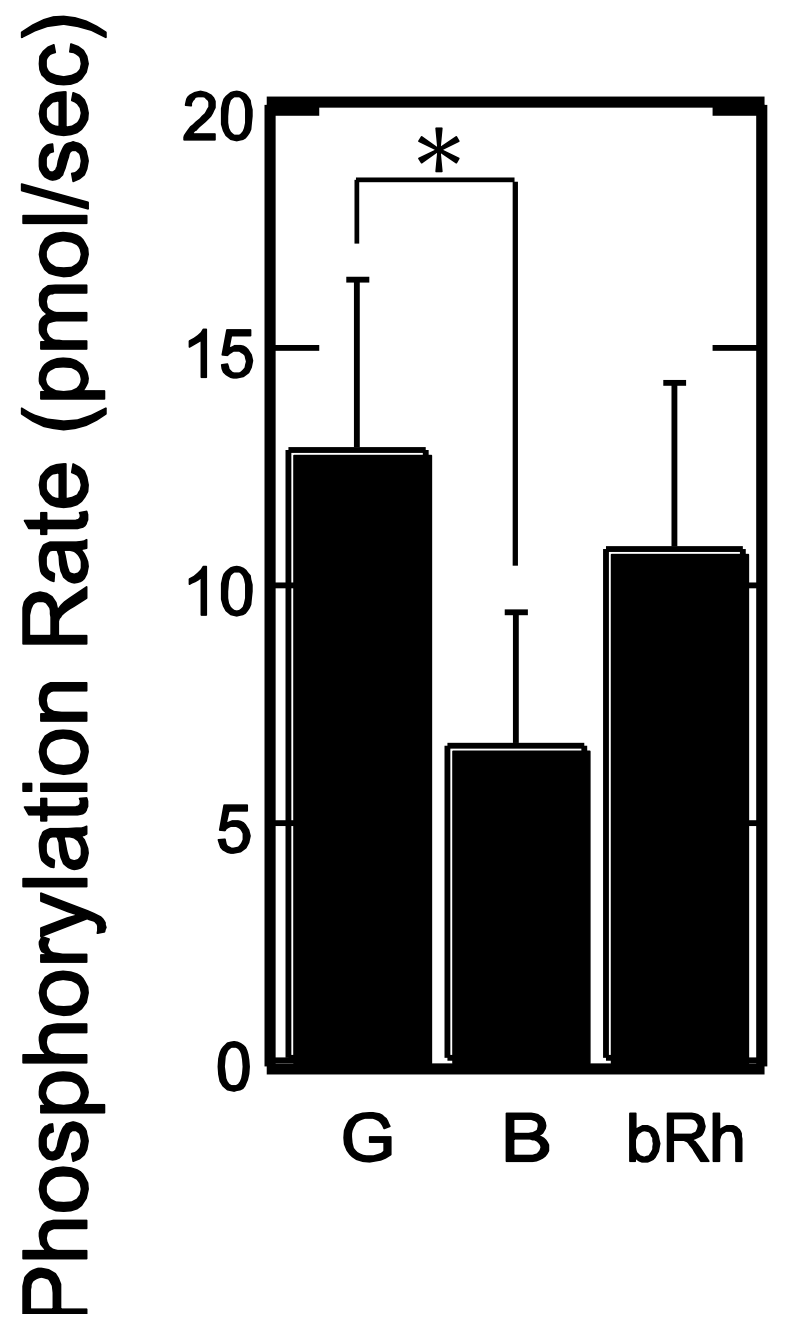

Figure 2. 


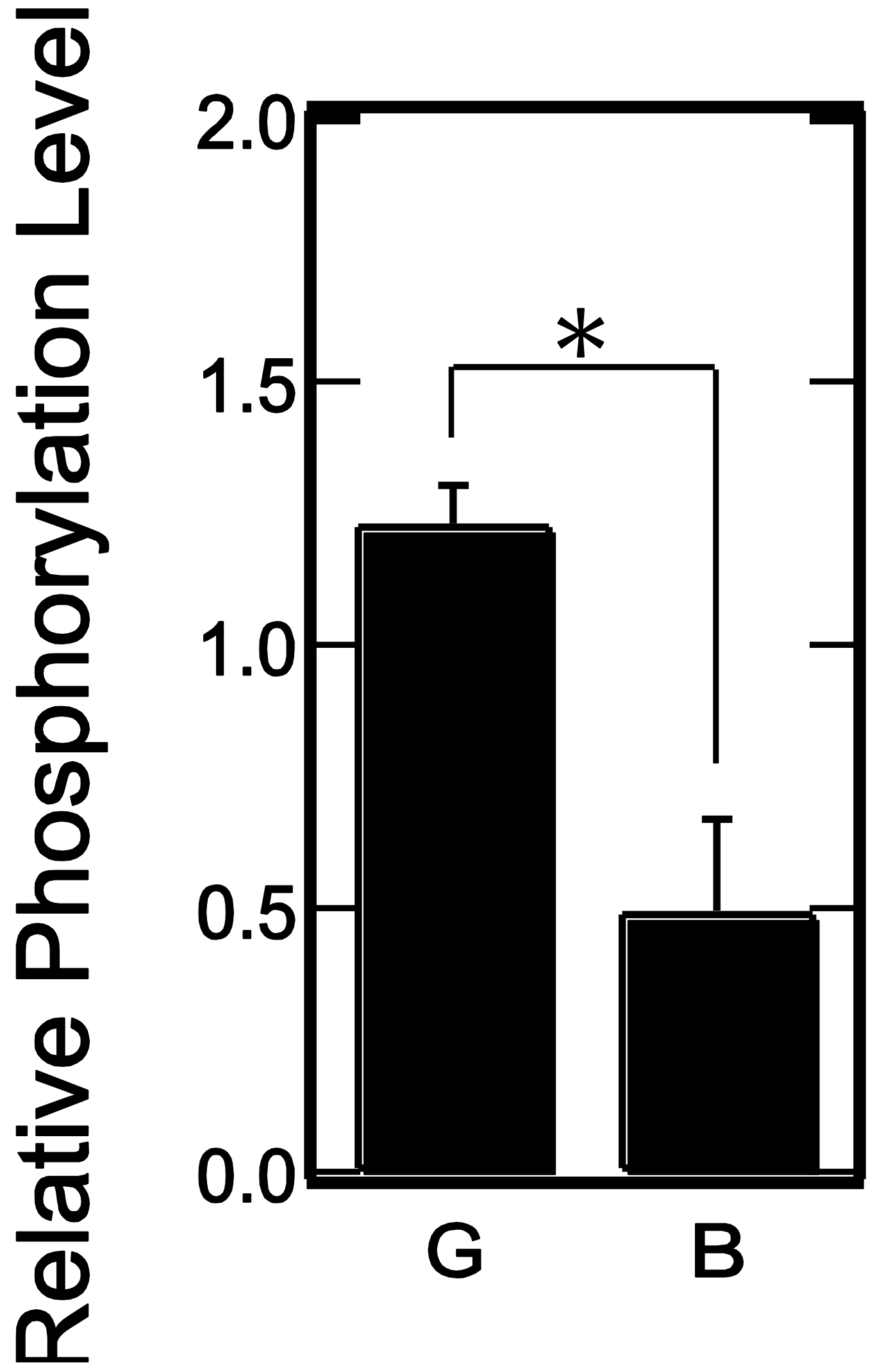

Figure 3. 